\title{
PFN-A - Bietet dieses neue Implantat zur Versorgung per/subtrochantärer Oberschenkelfrakturen Vorteile?
}

\author{
Th. J. Hockertz
}

Die Inzidenz der Femurfrakturen des koxalen Endes steigt parallel zur demografischen Entwicklung und steigenden Lebenserwartung. Es werden pro Jahr ca. 32500 Menschen mit per/subtrochantären Oberschenkelfrakturen versorgt. Dies entspricht einem Wirtschaftsvolumen unter DRG-Bedingungen von ca. 184 Millionen Euro in der Bundesrepublik Deutschland (Zahlenmaterial: Firma Lohmann + Birkner; Berlin).

Während für die 31 A1-Frakturen (ASIF/ AO-Klassifikation) mit der Dynamischen Hüftschraube seit Jahren ein adäquates Implantat zur Verfügung steht, waren die höhergradig instabilen A2- und A3Frakturen, insbesondere beim Vorliegen einer Osteoporose, immer als problematisch anzusehen.

Im letzten Jahrzehnt wurde die intramedulläre Stabilisierung dieser Frakturen zum Standardverfahren. Mit der breiten Markteinführung der zunächst nur mit einem singulären Kraftträger im Schenkelhals- und Hüftkopfbereich (z.B. Gammanagel) ausgestatteten Implantate
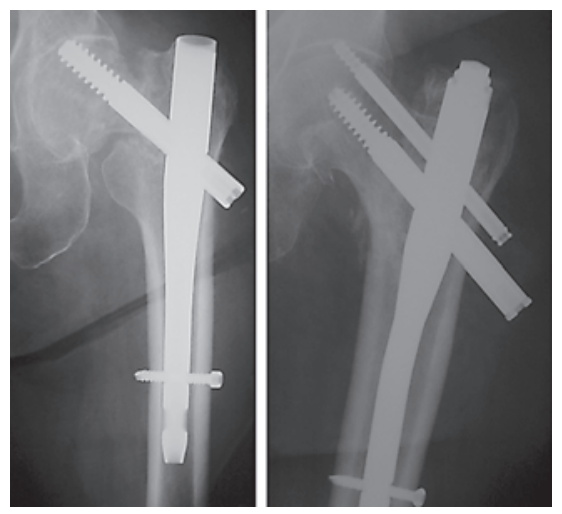

Abb.1 „Cut-off“ und „Z-Phänomen bei intramedullärer Stabilisierung.

OP-JOURNAL 2005; 21: 192-193

(c) Georg Thieme Verlag KG Stuttgart · New York

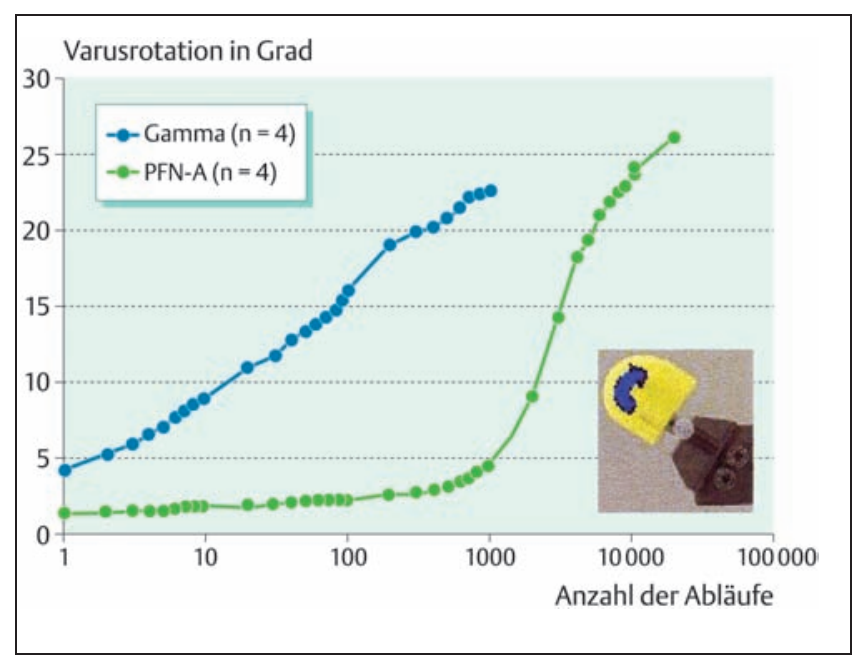

Abb. 2 Vergleichende Untersuchung bezüglich Rotationsstabilität und Varusdeformation im Schenkelhalsbzw. Hüftkopfbereich: PFN-A versus Gammanagel Quelle: LEGACY-Lab; Portland, USA.

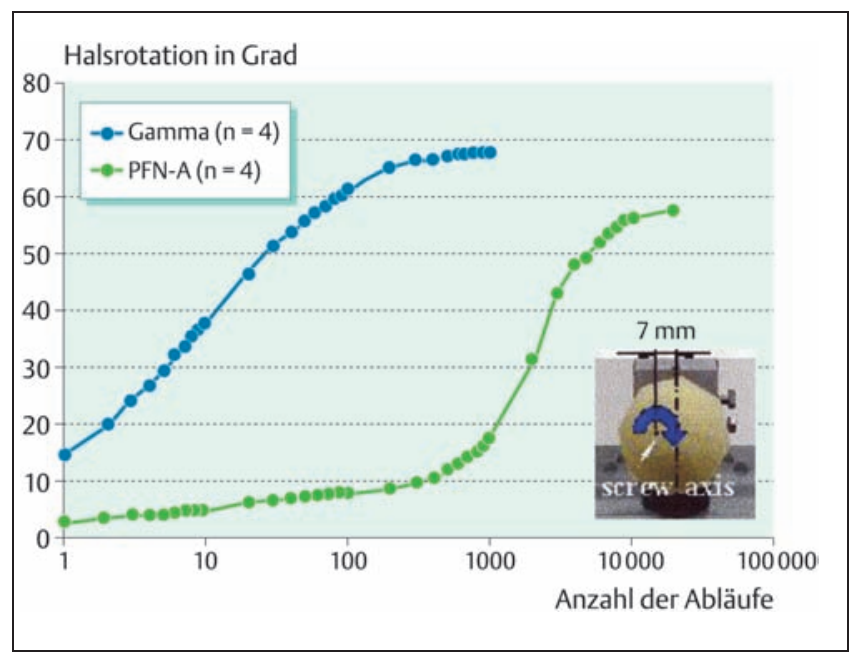

konnten viele der A2- und A3-Frakturen sicher zur Ausheilung gebracht werden.

Problematisch blieben die Mehrfragmentfrakturen sowie Frakturen bei höhergradiger Osteoporose. Das Auftreten einer primären/intraoperativen oder sekundären Rotationsfehlstellung im Bereich des Schenkelhalses konnte mit Einführung des PFN reduziert werden, dafür traten neue Probleme („Cut-off“ des Hüftkopfes oder „Z-Phänomen“) auf (Abb.1). Insbesondere die Verankerung von ge- windetragenden Kraftträgern im Hüftkopfbereich blieb problematisch.

Mit der Einführung des PFN-A will man der Lösung dieser Probleme näher kommen. Die wissenschaftliche Auswertung des an mehreren europäischen Zentren in klinischer Anwendung befindlichen Implantates findet bei Prof. Ch. Van der Werken (Utrecht, NL) statt.

Die Operationstechnik gleicht der des PFN. Die Eröffnung des Markraumes 


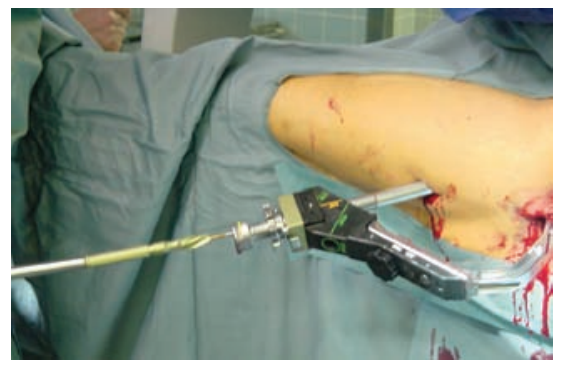

Abb.3 Einschlagen der Trageklinge über Führungsdraht und Zielinstrumentarium.

und Platzieren der Nagelkomponente bleibt im Wesentlichen unverändert. Im Gegensatz zum Eindrehen einer scharfkantigen Schraube findet hier ein Einschlagen einer sich frei drehenden Klinge in den Schenkelhals bzw. Hüftkopf Verwendung. Hierbei kommt es zu einer Spongiosaverdichtung um das Implantat und damit Verbesserung des Haltes im Knochen (Abb. 3).

In unserer Klinik findet dieses Implantat seit seiner Einführung im November 2003 bis November 2004 bei ca. 120 Menschen Verwendung. Bei einem Durchschnittsalter von über 80 Jahren ist der Anteil osteoporotischer Frakturen entsprechend hoch. Schwerwiegende intra-
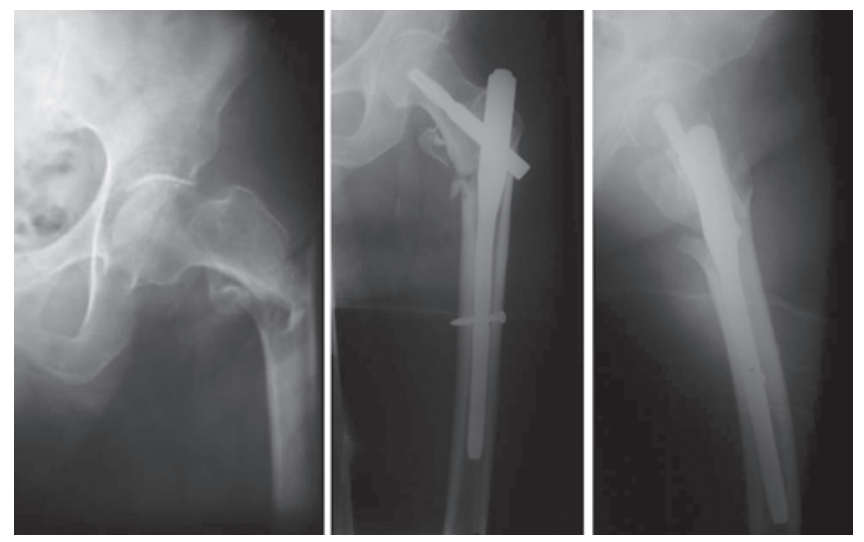

Abb. 4 Versorgung einer 31 A3-Fraktur mit PFN-A.

operative Komplikationen traten bislang nicht auf. Das Durchschneiden einer Klinge („Cut-off“) durch den Schenkelhals wurde bis jetzt ebenfalls nicht beobachtet. Bei 2 Patienten war eine offene Reposition erforderlich, ansonsten gelang die geschlossene Reposition. Es wurde bei zwei Patienten ein Klingenwechsel wegen eines zu weit nach lateral ausgetretenden Kraftträgers im postoperativem Verlauf durchgeführt. Ein Implantatversagen fand nicht statt.

Insgesamt zeichnet sich das neue Implantat durch hohe Bedienerfreundlichkeit bei Beachtung der OP-Anleitung und entsprechender Schulung aus. Das Implantat kann in minimalinvasiver OP-Technik eingebracht werden. Eine möglichst anatomische Reposition ist selbstverständlich weiter anzustreben. Die OP-Zeiten, im Zeitalter der DRGs und knappen Ressourcen sind immer wichtiger, erscheinen reduziert.

\section{Dr. med Thomas J. Hockertz}

Leitender Abteilungsarzt

Unfallchirurgische Klinik Städtisches Klinikum Braunschweig gGmbH

Holwedestr. 16

D-38118 Braunschweig 